\title{
Electrically Controlled Nano and Micro Actuation in Memristive Switching Devices with On-Chip Gas Encapsulation
}

\author{
Dean Kos, Hippolyte P. A. G. Astier, Giuliana Di Martino, Jan Mertens, Hamid Ohadi, \\ Domenico De Fazio, Duhee Yoon, Zhuang Zhao, Alexander Kuhn, Andrea C. Ferrari, \\ Christopher J. B. Ford, and Jeremy J. Baumberg**
}

\begin{abstract}
Nanoactuators are a key component for developing nanomachinery. Here, an electrically driven device yielding actuation stresses exceeding $1 \mathrm{MPa}$ with integrated optical readout is demonstrated. $10 \mathrm{~nm}$ thick $\mathrm{Al}_{2} \mathrm{O}_{3}$ electrolyte films are sandwiched between graphene and $\mathrm{Au}$ electrodes. These allow reversible room-temperature solid-state redox reactions, producing Al metal and $\mathrm{O}_{2}$ gas in a memristive-type switching device. The resulting high-pressure oxygen micro-fuel reservoirs are encapsulated under the graphene, swelling to heights of up to $1 \mu \mathrm{m}$, which can be dynamically tracked by plasmonic rulers. Unlike standard memristors where the memristive redox reaction occurs in single or few conductive filaments, the mechanical deformation forces the creation of new filaments over the whole area of the inflated film. The resulting on-off resistance ratios reach $10^{8}$ in some cycles. The synchronization of nanoactuation and memristive switching in these devices is compatible with large-scale fabrication and has potential for precise and electrically monitored actuation technology.
\end{abstract}

\section{Introduction}

Revolutions in nanotechnology rely on miniaturizing functional components, which is a particular problem for nanoactuators required to control nanostructures and manipulate matter

D. Kos, H. P. A. G. Astier, Dr. G. Di Martino, Dr. J. Mertens,

Dr. H. Ohadi, Prof. C. J. B. Ford, Prof. J. J. Baumberg

Cavendish Laboratory

University of Cambridge

J J Thomson Avenue, Cambridge CB3 OHE, UK

E-mail: jjb12@cam.ac.uk

D. De Fazio, Dr. D. Yoon, Dr. Z. Zhao, Prof. A. C. Ferrari

Cambridge Graphene Centre

9) J Thomson Avenue, Cambridge CB3 OFA, UK

Prof. A. Kuhn

Univ. Bordeaux

CNRS UMR 5255

Bordeaux INP

Site ENSCBP

33607 Pessac, France

The ORCID identification number(s) for the author(s) of this article can be found under https://doi.org/10.1002/smll.201801599.

DOI: 10.1002/smll.201801599 at the nanoscale. ${ }^{[1]}$ Several microactuators have been demonstrated based on electrostatic, ${ }^{[2]}$ electro-thermal, ${ }^{[2]}$ magnetic, ${ }^{[2]}$ and piezoelectric forces, ${ }^{[2]}$ as well as bimorphs. ${ }^{[3]}$ However, the transition to the nanoscale raises a number of fabrication and cost/complexity challenges. Smallscale bimorphs, such as carbon-nanotube/ aluminum nanoactuators, ${ }^{[4]}$ are hard to fabricate and position. Electroactive polymer films have been used for large-scale devices with areas of the order of $\mathrm{m}^{2}$, but they lack local control, and are complex to switch electrically. ${ }^{[5-7]}$ Organic molecules, such as DNA, can be folded into structures with chemically triggered actuating capabilities in liquid environments, ${ }^{[8]}$ but these are inherently slow (typically seconds). All these systems face challenges in fabrication and operation when device size is pushed towards the sub-100 nm scale, ${ }^{[9]}$ where high actuation stresses $(>1 \mathrm{MPa})$ are particularly important, since van der Waals interactions, capillary forces, and ionic charges become dominant.

Here we report an electrically driven nanoactuator based on memristive switches, which is compatible with large-scale fabrication and incorporates optical readout capabilities. Memristive devices have been proposed by Ref. [10] and demonstrated in Ref. [11], and can be obtained with thin layers of oxides, as well as protein-based materials, ${ }^{[12,13]}$ by electrically inducing a reversible local change in conductivity via ion migration at the nanoscale. This ion migration triggers a redox reaction that is often accompanied by gas release from the oxide, which is usually fatal for the memristive switch. ${ }^{[14-16]}$ In our work, we instead trap this gas in micrometer-sized tanks to create an electrically driven reversible actuator based on memristive switching.

\section{Results and Discussion}

The functional component of the actuator is a thin $\mathrm{Al}_{2} \mathrm{O}_{3}$ film, sandwiched between a graphene electrode and a flat $\mathrm{Au}$ substrate. The top single layer of graphene (SLG) is grown by chemical vapor deposition (CVD) ${ }^{[17]}$ (see the Supporting 

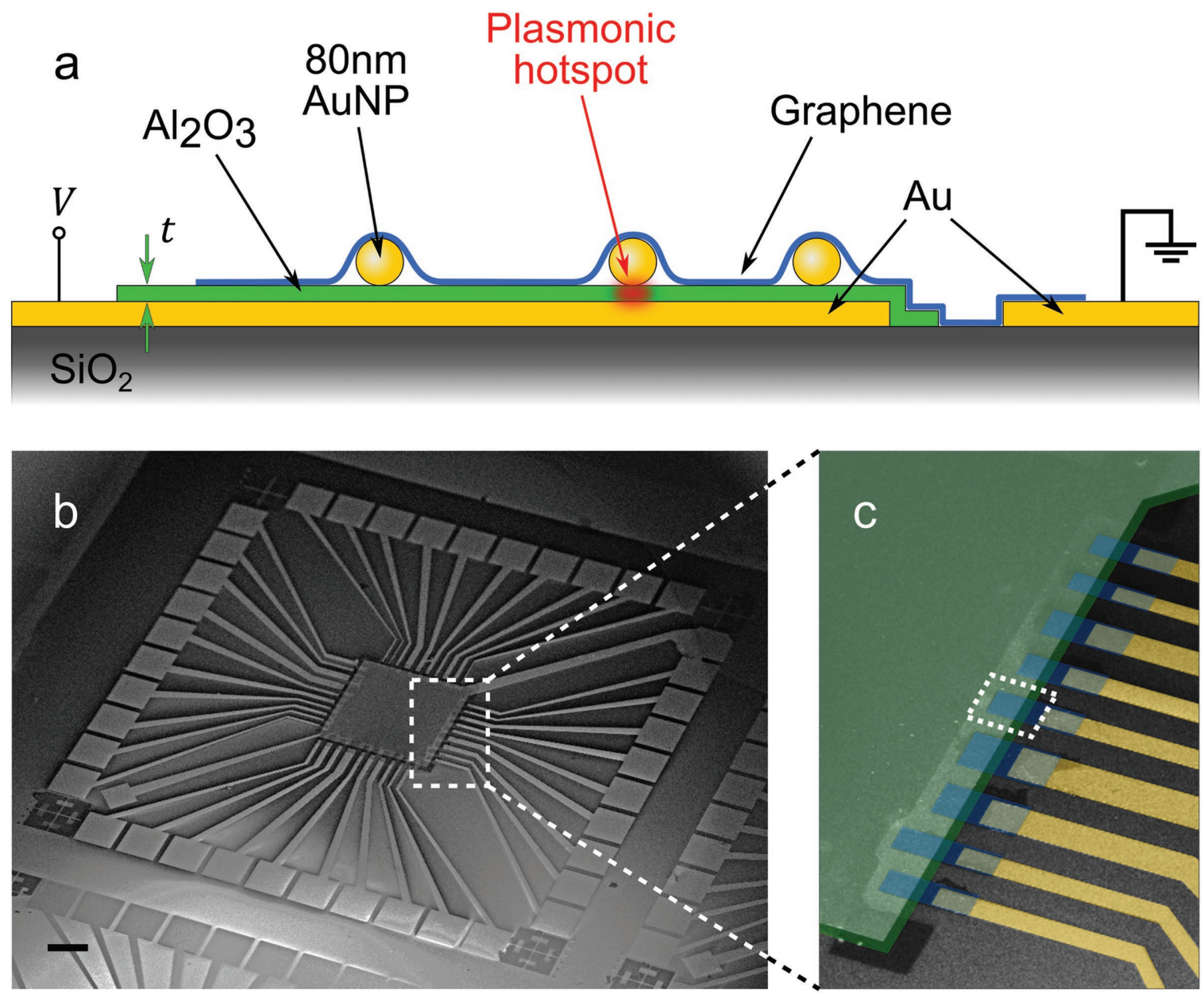

Figure 1. Device design. a) The $\mathrm{Al}_{2} \mathrm{O}_{3}$ layer $(t \approx 8$ or $12 \mathrm{~nm})$ is sandwiched between a separately contacted top SLG and bottom Au substrate to which a potential $V$ is applied. Low-density $80 \mathrm{~nm}$ Au NPs under the SLG allow optical tracking. b,c) SEM images of device with Au fingers (false color yellow) contacting the SLG pads (blue) sitting on $\mathrm{Al}_{2} \mathrm{O}_{3}$ (green). Scale bar: $200 \mu \mathrm{m}$.

Information), while the thin $\mathrm{Al}_{2} \mathrm{O}_{3}$ film (thickness $t \approx 12$ or $8 \mathrm{~nm}$ ) is deposited via atomic layer deposition (ALD) on the 100-nm-thick bottom gold contact (Figure 1a; also see Experimental Section). By incorporating gold nanoparticles (AuNPs) under the SLG in a nanoparticle-on-mirror (NPoM) geometry, ${ }^{[18,19]}$ we optically track the state of the thin $\mathrm{Al}_{2} \mathrm{O}_{3}$ film under bias. Incident light is trapped in the plasmonic hotspot tightly localized within the nanoscale gap between AuNP and Au substrate, providing optical access to changes in the thin film morphology and its dielectric properties. ${ }^{[20]}$ Because Au NPs can be deposited over areas of several $\mathrm{cm}^{2}$ and their optical response is reproducible, we optically probe large regions of the thin film in real time. The NPs are drop-cast over the surface with a number density controlled by the deposition time, and optimized to ensure light collection for spectroscopy from individual NPs within the collection spot.

The SLG and Au contacts are arranged as independent junctions (Figure 1b,c) electrically isolated from each other so that a voltage $V$ can be applied across independent $\mathrm{SLG}-\mathrm{Al}_{2} \mathrm{O}_{3}-\mathrm{Au}$ structures. SLG is used as the top electrode for its conductivity, ${ }^{[21]}$ transparency (making the encapsulated NPs optically accessible), ${ }^{[21]}$ flexibility (conforming to mechanical deformations in the underlying structure), ${ }^{[21]}$ and impermeability to gases. ${ }^{[21]}$ Raman spectroscopy is used to monitor the presence of defects, ${ }^{[22]}$ doping, ${ }^{23,24]}$ and strain $^{[25-28]}$ (see the Supporting Information). Using $\approx 1-100 \mathrm{~cm}^{2}$ CVD SLG sheets and optical lithography allows parallel fabrication of a number of junctions.

Electrical measurements are used to characterize the junctions at room temperature. The initial state is insulating with conductance in the $0.1-10 \mathrm{pS}$ range for areas $\approx 500 \mu \mathrm{m}^{2}$ and $t \approx 8 \mathrm{~nm}$. The overlap region between $\mathrm{SLG}$ and $\mathrm{Al}_{2} \mathrm{O}_{3}$ with a junction area $\approx 500 \mu \mathrm{m}^{2}$ corresponds to a conductance

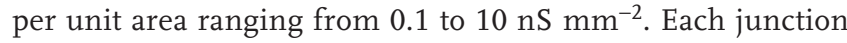
can be made conductive (switched on) by applying a critical voltage across it. In the case of devices with $8 \mathrm{~nm} \mathrm{Al}_{2} \mathrm{O}_{3}$ layers, this voltage is $\approx 5 \mathrm{~V}$ (for $12.5 \mathrm{~nm}$ thick layers devices switch on at $\approx 7 \mathrm{~V}$ ). The turn-on transition appears as a sudden jump in current, in which the conductance rises to values in the $1-100 \mu \mathrm{S}$ range, which for a typical junction area of $500 \mu \mathrm{m}^{2}$ corresponds to a conductance per unit area $1-100 \mu \mathrm{S} \mathrm{mm} \mathrm{mm}^{-2}$. In a given cycle, on-off resistance ratios as high as $10^{8}$ are observed (Figure 2a,b), which compare favorably to some of the highest ratios in memristors. ${ }^{[29-32]}$ Reversing the voltage 

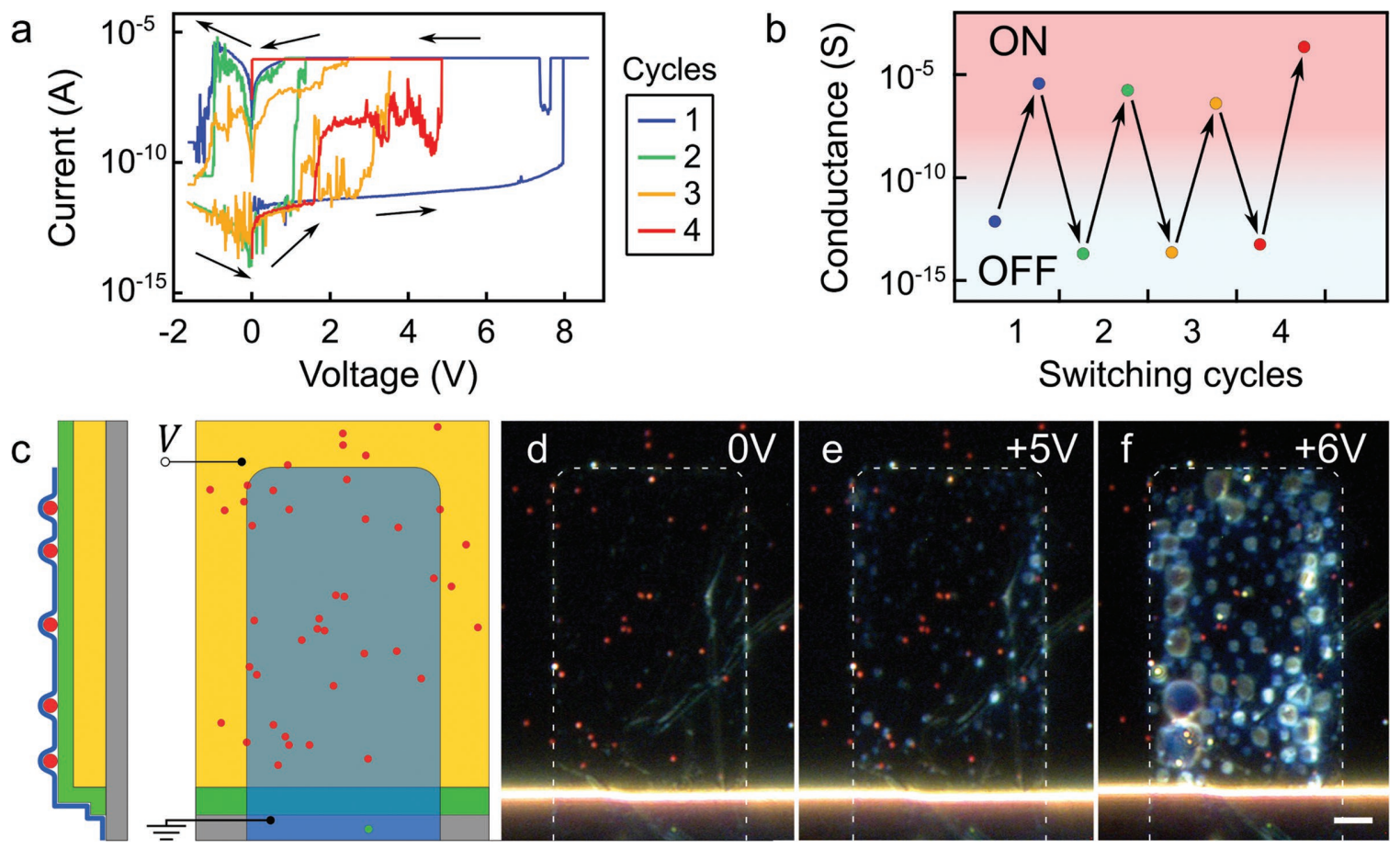

Figure 2. Device dynamics under applied potential. a) $I-V$ plot of transitions between on and off states driven by varying junction voltage to positive or negative polarities. Magnitude of current $|l|$ shown on logarithmic scale; legend color indicates order in which the measurements are taken, while a current compliance limits the maximum current. b) Junction conductance in on and off states for cycles shown in (a). c) Single-device finger with SLG (blue) on $8 \mathrm{~nm} \mathrm{Al}_{2} \mathrm{O}_{3}$ (green) thin film on Au back contact. Low-density $80 \mathrm{~nm}$ Au NPs (red) are sandwiched between $\mathrm{SLG}_{\text {and }} \mathrm{Al}_{2} \mathrm{O}_{3}$. Dark-field images obtained with application of d) $0 \mathrm{~V}$, e) $5 \mathrm{~V}$, and f) $6 \mathrm{~V}$. Strong light scattering is observed for increasing voltages. Scale bar: $5 \mu \mathrm{m}$.

polarity across the junction drives the opposite reaction and decreases the conductance back towards the initial state, seen either in a sudden drop in current or in a more gradual transition (Figure $2 \mathrm{a}$ ). We note $\approx 5$ cycles can be switched in this way (Figure 2b), before the memristive behavior is compromised. In either state, the junction remains stable when varying the voltage within a small range, between -1 and $1 \mathrm{~V}$. At $0 \mathrm{~V}$ the junctions are stable for tens of hours. However, sometimes a slight decrease in conductivity is observed. After typically 5-10 cycles, the conduction state can no longer be changed with voltage.

We perform further electrical measurements while optically imaging the SLG from the top (Figure 2c). When a field exceeding $5 \times 10^{8} \mathrm{~V} \mathrm{~m}^{-1}$ is applied $\left(V_{t} \approx 5 \mathrm{~V}\right.$ for $t \approx 8 \mathrm{~nm}$, $V_{t} \approx 7 \mathrm{~V}$ for $t \approx 12 \mathrm{~nm}$ ), we observe a strong scattering arising from different regions of the SLG pad (Figure 2d-f). These bright regions (which as we show below are caused by microbubbles) expand faster as $V$ is further increased, and merge into fewer larger regions as they expand more. The AuNPs within the bright regions change the color from red to yellow (Figure 3a,b), signaling a modification of the NPoM gap. Such behavior is consistently observed for all NPs. The NPoM geometry is thus helpful to investigate the real-time dynamics of such thin films or bubbles.

The formation of each bubble results in a blueshift of the NP-coupled mode seen in dark-field scattering spectroscopy (Figure 3c,d), together with a simultaneous increase in current (Figure 3e). Reversing the voltage causes the bubbles to gradually shrink, enabling voltage-driven height manipulation. Complete bubble deflation can be achieved, fully recovering the initial NP spectrum (Figure 3f,g). Again the switching process in these devices can be repeated $\approx 5$ times (Figure $3 \mathrm{f}, \mathrm{g}$ ). As discussed below, the failure occurs if the bubbles collapse enough after inflation to allow $\mathrm{O}_{2}$ escape from reservoirs at the edge of the $\mathrm{Al}_{2} \mathrm{O}_{3}$. In a given junction, the location and relative size of the bubbles when a device is first turned on are random, and a typical statistical distribution of bubble heights is shown in Figure S2 (Supporting Information). However, the location, size, and height of the bubbles are maintained in successive switching cycles when using the same electrical parameters, enabling the NPs on bubbles to undergo repeatable transitions (Figure $3 \mathrm{~g}$ ). Once the voltage is turned off, the position and size of the bubbles remain stable for months.

The NP blueshifts can arise from three different scenarios: refractive index changes in the medium surrounding the NP (scenario 1), alterations in spacer conductivity (2), or variations in distance from the mirror, where it is sensitive to nanometer displacements (3). Scenario 1 requires an unfeasibly high refractive index variation (in excess of $\Delta n=0.4$ ) to account for a $50 \mathrm{~nm}$ shift. ${ }^{[18]}$ Variations in conductivity can induce a shift, due to shorting of the optical charge oscillations between NP and substrate, ${ }^{[33]}$ but requires a much larger increase in $\mathrm{Al}_{2} \mathrm{O}_{3}$ conductivity than measured. Similarly, a vertical displacement of the NP (3) could cause a shift of the coupled mode resonance ${ }^{[34]}$ needing the NP to be moved away from the mirror to induce the observed blueshift. Proving this latter scenario thus requires morphological analysis of the surface.

Angled scanning electron microscopy (SEM) images of the bubbles reveal an inflation of the device surface with the 

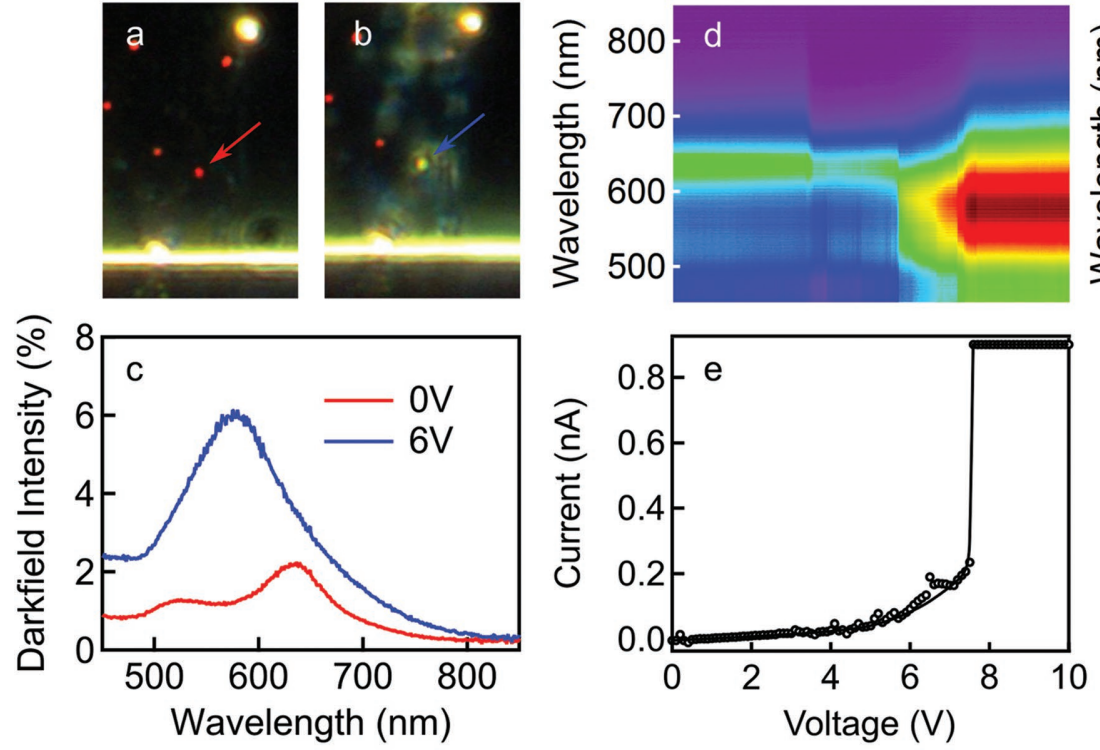

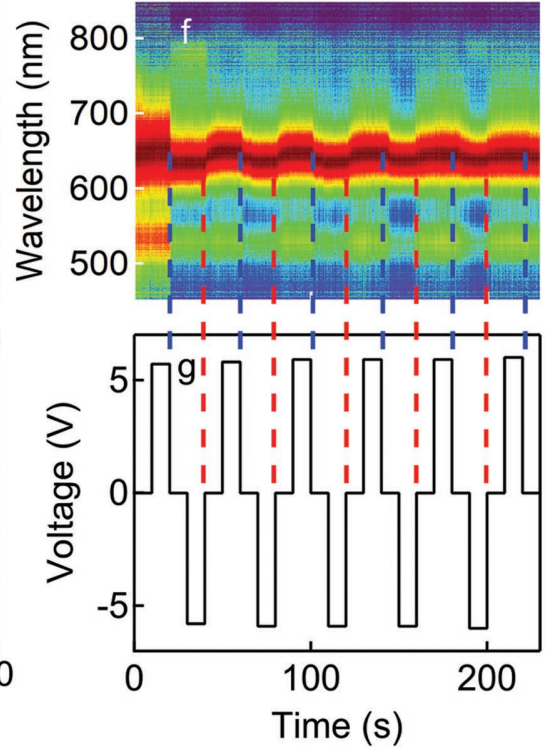

Figure 3. Optical evolution with applied potential. a,b) NP color switching in dark field imaging, with c) corresponding scattering spectra from this NP. d) NP scattering spectrum for increasing voltages with e) corresponding measured current (line is guide to the eye). f) Scattering spectra when plasmon resonance switches under voltage cycling $-V \leftrightarrow+V$ over the time sequence shown in (g). Positive voltages trigger blueshifts while negative ones promptly recovers the initial condition.

AuNPs sitting on top (Figure 4a), confirming scenario 3. Atomic force microscopy (AFM) topography measurements are used to quantify the size of the larger bubble reservoirs (visible in dark-field microscopy), which can reach up to tens of micrometers in diameter and up to $1 \mu \mathrm{m}$ in height (Figure 4b,c, see also the Supporting Information). The bubbles observed in dark-field microscopy thus correspond to vertical displacements of the device surface, which match the blueshift in the NPoM scattering spectra. ${ }^{[34]}$ In the early stage of inflation, bubble actuation can only be detected in real time through the NP scattering spectra, sensitive to sub-nanometer displacements (see discussion in the Supporting Information). The additional rise in scattering intensity of the NPoMs (Figure 3c) is caused by interference effects when the distance exceeds $100 \mathrm{~nm},{ }^{[35]}$ which agrees with the height range measured using AFM.

The reservoir inflation could arise from several types of deformation. Bulging of the Au bottom contact due to electrostatic forces between the electrodes is ruled out since bubble formation is not observed for negative applied voltages, although electrostatic forces should be independent of voltage polarity. SLG corrugation alone would not cause the bright scattering regions observed in dark-field, because the SLG itself is barely visible in microscopy. The AuNPs play no active role, since the initial position of the bubbles is unrelated to the NP positions. We conclude that changes in the $\mathrm{Al}_{2} \mathrm{O}_{3}$ layer are therefore responsible for reservoir inflation.

Thin $\mathrm{Al}_{2} \mathrm{O}_{3}$ films are known to allow migration of oxygen ions, ${ }^{[36]}$ and have widely been used in resistive switching devices. ${ }^{[14,37-40]}$ In such devices, a voltage applied across an oxide layer such as $\mathrm{TiO}_{2}$ or $\mathrm{Al}_{2} \mathrm{O}_{3}$ drives $\mathrm{O}^{2-}$ ions towards one of the electrodes. This leaves oxygen vacancies in the material, doping the oxide and changing the resistivity of the layer, thus making it suitable for resistive switching applications. In the case of $\mathrm{TiO}_{2}, \mathrm{O}^{2-}$ ion migration and vacancy formation have been confirmed by spatially resolved X-ray spectromicroscopy. ${ }^{[41]}$ When using inert Pt electrodes, the ions can then oxidize upon
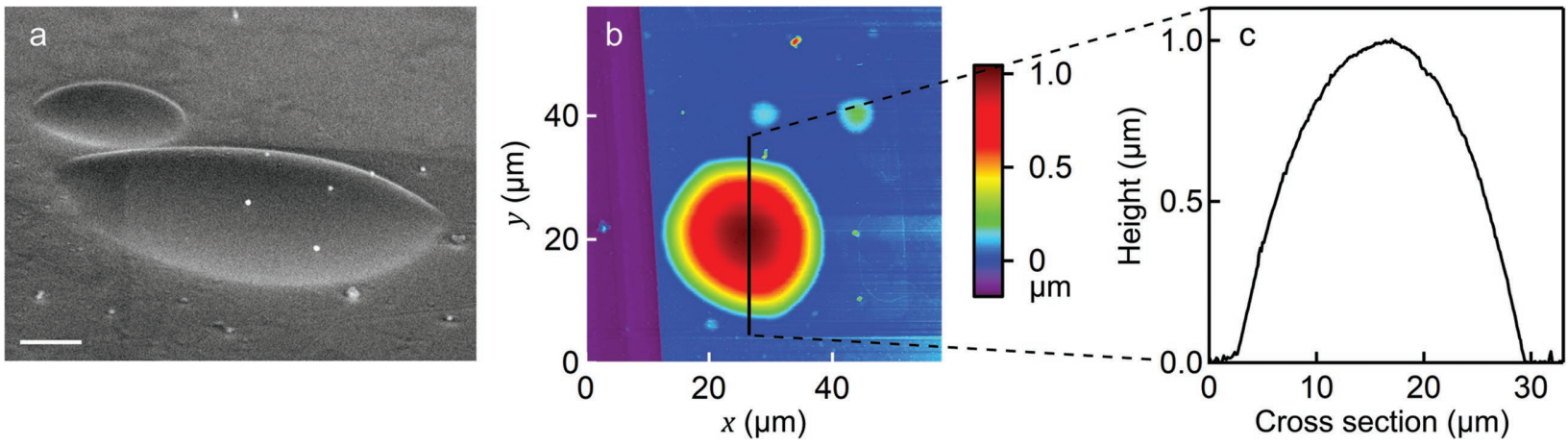

Figure 4. Bubble reservoir characterization. a) SEM image of inflated reservoir, with NPs sitting on top. Scale bar: $1 \mu \mathrm{m}$. b) AFM topography image with c) cross section showing reservoir height along the line. 
reaching the positive electrode via the reaction $2 \mathrm{O}^{2-} \rightarrow 4 e^{-}+$ $\mathrm{O}_{2(\mathrm{~g})}$, forming bubbles of oxygen gas. ${ }^{[16]}$ Until now however, bubbles have only persisted while the voltage is applied, rapidly disappearing once the bias is removed. Pt and $\mathrm{Au}$ are common choices for the inert electrode materials in this type of memristor, since they have good conductivity and are not affected by oxidation. There is no evidence that Au plays a catalytic role in the redox reaction occurring during switching. ${ }^{[42]}$ Modifications in the density of oxygen vacancies under applied voltage have also been studied in $\mathrm{NiO}$ films, where growth of a metallic $\mathrm{Ni}$ phase has been proposed as the switching mechanism, ${ }^{[15]}$ although with no direct evidence in working devices. ${ }^{[43,44]}$ The exact oxide reduction mechanisms are therefore still unclear. Studying these in typical resistive switching devices based on planar metal/oxide/metal junctions is problematic as they prevent in situ access for optical or electron microscopies.

Our devices share the same structure as such resistive switches. We conclude that reservoir inflation is similarly caused by drift of $\mathrm{O}^{2-}$ ions which move towards the positive electrode, there converting into oxygen gas. Our data thus confirm the observation of oxygen bubbles reported so far only for $\mathrm{TiO}_{2},{ }^{[16]}$ consistent with high-angle energy-dispersive X-ray spectroscopy (EDX). The dynamics of the reservoirs and the increased expansion speed with higher voltage also agree with previous reports. ${ }^{[16]}$ The currently accepted view of the resistive switching mechanism in valence-change cells is that the high-conductivity state is due to a localized conductive filament, ${ }^{[45]}$ but its composition and structure have not yet been directly observed in situ. Formation of these filaments has often been associated with local deformation of the device in the form of crater-like structures, ${ }^{[46]}$ and this seems consistent with the formation of a gas reservoir bursting due to excessive pressure. In our devices, conduction through the $\mathrm{Al}_{2} \mathrm{O}_{3}$ film starts at a site where $\mathrm{O}^{2-}$ ion conductivity is likely higher than the surroundings. Rapidly, this local migration of $\mathrm{O}^{2-}$ ions forms $\mathrm{O}_{2}$ gas at the Au electrode which lifts the graphene $/ \mathrm{Al}_{2} \mathrm{O}_{3}$ film away from the $\mathrm{Au}$, interrupting the conduction path. Conduction can then only occur through the migration of $\mathrm{O}^{2-}$ at the boundaries of the reservoir via new migration paths formed in the $\mathrm{Al}_{2} \mathrm{O}_{3}$ film, thereby continuously producing more $\mathrm{O}_{2}$ gas and enlarging the reservoir laterally (Figure 5a).
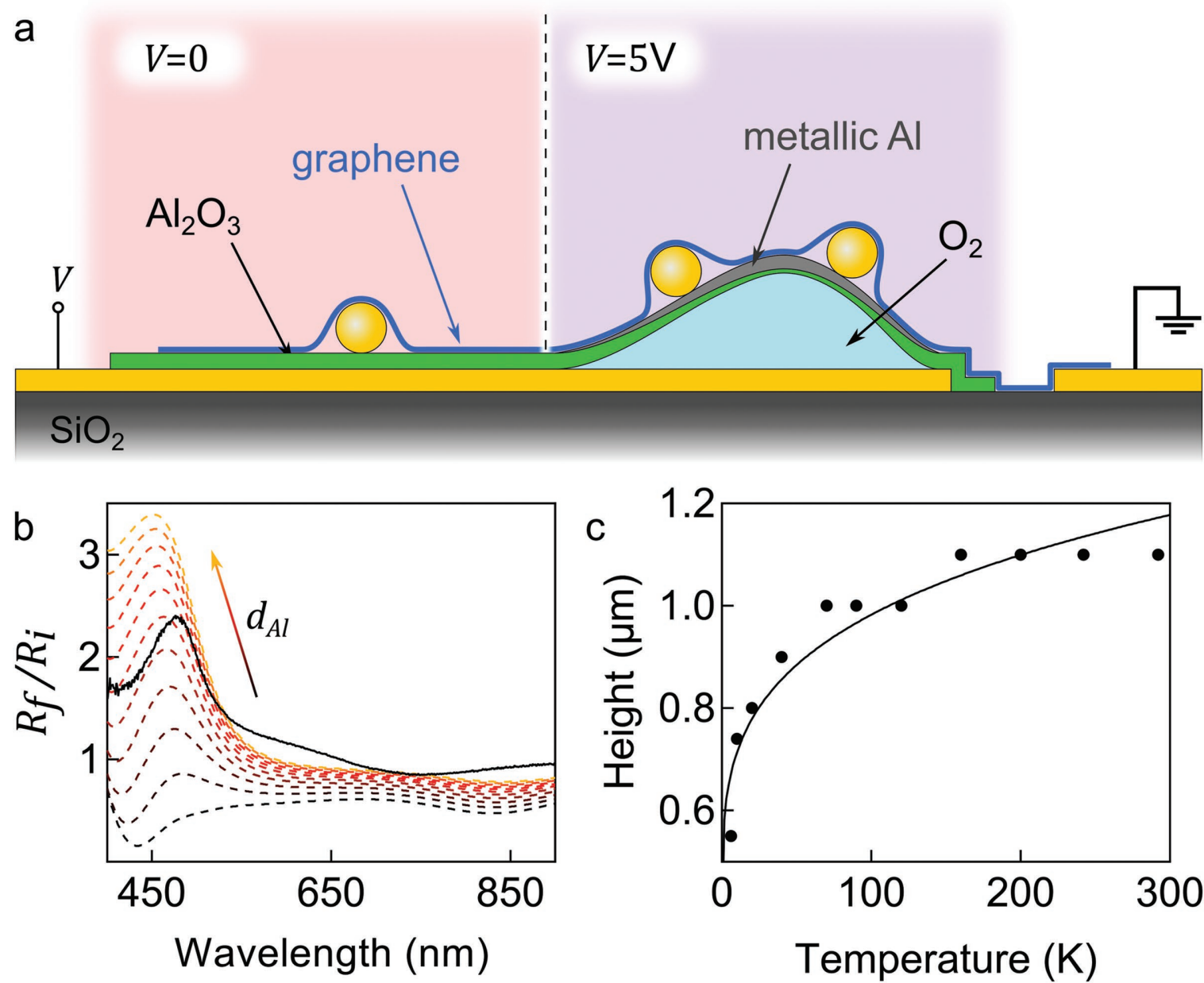

Figure 5. $\mathrm{Al}_{2} \mathrm{O}_{3}$ redox transformation into $\mathrm{Al}$ and $\mathrm{O}_{2}$. a) Thin $\mathrm{Al}_{2} \mathrm{O}_{3}$ film $\left(\mathrm{V}=0\right.$ ) separates into metallic $\mathrm{Al}$ and $\mathrm{O}_{2}$ gas $(\mathrm{V}>5 \mathrm{~V})$ with a growing bubble reservoir. b) Ratio of measured reflectivity after $\left(R_{f}\right)$ and before $\left(R_{i}\right)$ reservoir inflation (solid line), compared to the thin film model (dashed) with increasing Al thickness $d_{A l}$, identifying $d_{A l} \approx 10 \mathrm{~nm}$, and predicting the same inflation height as that found from AFM. c) Bubble height decreases as temperature is lowered, due to decreasing volume of internal $\mathrm{O}_{2}$. Fit (line) shows ideal gas dependence $h \propto T^{1 / 4}$. 
A corresponding solid-state electrochemical reaction is then driven at the graphene electrode, $\mathrm{Al}^{3+}+3 e^{-} \rightarrow \mathrm{Al}^{0}$ producing a thin metallic Al film. Rather than localized conductive filaments, found in most other memristors, which would be unfavorable to grow the bubble, this scenario suggests a uniform Al film forming near the surface of the reservoir.

To confirm this picture, we compare the reflectivity measurements on a bubble region before and after reservoir inflation. The SLG electrode in our devices is crucial for enabling this real-time optical tracking of the redox underlayer. We collect initial bright-field reflectivity spectra $R_{i}$ before any voltage is applied on the top of each bubble with a $50 \times$ objective, to focus broadband white light which is confocally collected at a detection fiber. After forming the reservoir, the modified reflectivity $R_{f}$ is measured, and $R_{f} / R_{i}$ is plotted to highlight the spectral changes. We compare $R_{f} / R_{i}$ with transfer matrix calculations using a multilayer model of the inflated reservoir structure ${ }^{[47]}$ (Figure $5 b$ ). By fitting the model parameters (thickness of metallic Al film, thickness of residual $\mathrm{Al}_{2} \mathrm{O}_{3}$ layer, and height of gas reservoir; see Section S3, Supporting Information) to the experimental data, we find a complete disagreement unless a metallic Al film is present. Optimizing the fits resolves an Al thickness of $10 \mathrm{~nm}$, with $\mathrm{Al}_{2} \mathrm{O}_{3}$ residual film of $4.5 \mathrm{~nm}$, and reservoir height of $165 \mathrm{~nm}$ (in good agreement with the $175 \mathrm{~nm}$ average height from AFM for bubbles of this size; Figure S3, Supporting Information). Similar reflectivity changes are seen on other bubbles. This optical modeling also confirms that the Al nanolayer separates from the Au substrate, storing the oxygen gas between them.

The presence of gas is further demonstrated by cooling down and optically tracking the changing size of a $1 \mu \mathrm{m}$ bubble from 292 to $6 \mathrm{~K}$ in situ. Images taken at different temperatures are used to infer the bubble height by fitting the interference fringes from light reflected off the surface of the bubble and off the bottom Au substrate with a simple model (Section S1, Supporting Information). We observe a stronger decrease in reservoir height as the temperature is lowered below $150 \mathrm{~K}$ (Figure $5 \mathrm{c}$ ), reducing by about a factor of two, and matching the ideal gas law prediction $h \propto T^{1 / 4}$ (liquefaction is not achieved at these pressures; see also Section S2, Supporting Information). The shape and size of the reservoir are fully restored when the sample is heated back to $292 \mathrm{~K}$, suggesting that temperature control can also be used for nanoactuation and height finetuning, in addition to electrical driving. We exclude the presence of water in the devices since the ALD growth occurs at $180{ }^{\circ} \mathrm{C}$, and we do not observe any freezing signature at $0{ }^{\circ} \mathrm{C}$. To further prove the containment of pressurized gas, the tip of an AFM probe is used to pierce a hole in the surface of an inflated bubble, resulting in immediate deflation.

Given the presence of gas, we can infer its pressure from the geometrical parameters of each bubble and the material properties of its walls (Section S2, Supporting Information). The strain in the bubble surface film is found from ${ }^{[48]}$ $\varepsilon=2 h^{2} / 3 a^{2}$, where $h$ is the bubble height and $a$ its base radius, and ranges from $0.35 \%$ to $1 \%$ depending on the aspect ratio of the bubble, corresponding to a stress level well below the ultimate tensile strength of either graphene ${ }^{[49]}$ or $\mathrm{Al}_{2} \mathrm{O}_{3} \cdot{ }^{[50]}$ Raman spectroscopy is used to measure the strain in SLG ${ }^{[2,26,51]}$ for a similar bubble. Comparing to the deflated junctions yields a shift (Figure S7, Supporting Information) corresponding to a strain $\approx 0.1 \%$ (Sections S2 and S8, Supporting Information), consistent with the estimate for the laminate $\mathrm{SLG} / \mathrm{AL}_{2} \mathrm{O}_{3}$ film. Such strain values imply typical internal bubble pressures in the range of $20 \pm 10 \mathrm{~atm}$ (Section S2, Supporting Information). We find the amount of oxygen required to give this pressure is indeed a sizeable fraction of that locked up in the $\mathrm{Al}_{2} \mathrm{O}_{3}$ film ( $\approx 60 \%$, see Section S2, Supporting Information). Generally, smaller bubbles have higher internal actuation stress, thus likely requiring an overpotential to initially drive the reaction. As an estimate, a typical current of $1 \mathrm{nA}$ applied for $1 \mathrm{~min}$ across a junction would correspond to the migration of $10^{11} \mathrm{O}^{2-}$ ions. This matches a similar estimate of the number of oxygen atoms trapped as $\mathrm{O}_{2}$ in the bubbles assuming a pressure of $20 \mathrm{~atm}$ and typical bubble sizes after inflation. This is thus consistent with oxygen migration being a major conduction mechanism in our film. Furthermore, it explains the failure mechanism of the devices, which is most likely caused by localized damage to one of the encapsulating layers (SLG and $\mathrm{Al}_{2} \mathrm{O}_{3}$ ), that allows $\mathrm{O}_{2}$ to escape during switching and thus inhibits the inverse reaction. Strain probably plays a key role in the process. Thus, reducing it during actuation or increasing the encapsulating layer stiffness would improve device cyclability. A method to realize this is employing multilayer instead of SLG as top electrode, and the use of a surface treatment on top of the $\mathrm{Al}_{2} \mathrm{O}_{3}$ to reduce friction and aid conformal deformation of SLG to the surface. For bubble inflation, application of a low voltage $\left(\approx 5-7 \mathrm{~V}\right.$ depending on $\mathrm{Al}_{2} \mathrm{O}_{3}$ thickness) for a long time rather than a high voltage $(>10 \mathrm{~V})$ for a short time also leads to better cyclability.

At room temperature and low voltage, we are able to reversibly convert $>60 \%$ of thin $\mathrm{Al}_{2} \mathrm{O}_{3}$ films to metallic $\mathrm{Al}$ and back on demand. Our choice of electrode/electrolyte materials, forming chemically stable $\mathrm{SLG} / \mathrm{Al} / \mathrm{Al}_{2} \mathrm{O}_{3}$ and $\mathrm{Au} / \mathrm{O}_{2}$ interfaces, is what allows the reservoirs to remain stable for months when the bias is removed. A negative voltage triggers the oxygen to recombine with the $\mathrm{Al}$, thus reforming the $\mathrm{Al}_{2} \mathrm{O}_{3}$ film. This depends on the impermeability of SLG to $\mathrm{O}_{2},{ }^{\left[{ }^{[1]}\right.}$ while the strength of the $\mathrm{SLG} / \mathrm{Al} / \mathrm{Al}_{2} \mathrm{O}_{3}$ film allows $\mathrm{O}_{2}$ gas to be locally stored at high pressures without rupturing its flexible container. The internal pressure can be tuned by choice of layer thicknesses.

\section{Conclusion}

We have shown that SLG/Au electrodes combine with solidstate electrolyte thin films of $\mathrm{Al}_{2} \mathrm{O}_{3}$ to produce nanoactuators, capable of yielding high stress, which are electrically controlled. The applied electrical field drives $\mathrm{O}^{2-}$ drift, creating local chemical energy storage in the $\mathrm{Al}$ metal and $\mathrm{O}_{2}$ gas which can be triggered to recombine, thereby deflating the actuator. This device geometry has much in common with memristive switches but with an optically accessible electrode and provides new insights into the switching mechanisms. Graphene electrodes improve the reliability and resilience of this resistive switching. Reversible redox of $\mathrm{Al}_{2} \mathrm{O}_{3}$ is possible at room temperature under benign conditions creating high-pressure oxygen micro-fuel reservoirs under graphene encapsulation. Plasmonic rulers 
allow the dynamics of this process to be studied in real time. Both reactive species $\left(\mathrm{Al}\right.$ and $\left.\mathrm{O}_{2}\right)$ are stored in proximity for months. This promises a wide range of applications in nano and micro actuation and local energy storage in pressurized reservoirs, while allowing integration with current thin film technologies.

\section{Experimental Section}

Sample Preparation: Device fabrication was started with evaporation of a $20 \mathrm{~nm} \mathrm{Ti}$ adhesion layer, followed by $100 \mathrm{~nm}$ of Au deposited by evaporation onto a 285-nm-thick $\mathrm{SiO}_{2}$ on $\mathrm{Si}$ substrate in a pattern defined using optical lithography. The $\mathrm{Al}_{2} \mathrm{O}_{3}$ layer was deposited by ALD, without any prior adhesion layer, and using 60- and 90-layer deposition cycles to provide different thicknesses for comparison. The ALD was done at a temperature of $180^{\circ} \mathrm{C}$ in a vacuum chamber, which ensures a water-free $\mathrm{Au}-\mathrm{Al}_{2} \mathrm{O}_{3}$ interface. The $\mathrm{Al}_{2} \mathrm{O}_{3}$ layer was subsequently patterned via optical lithography and chemically etched using Microposit MF-319. AuNPs (80 nm diameter) from BBI Solutions were drop-cast on the substrate to obtain a number density of $\approx 0.1 \mu \mathrm{m}^{-2}$ on the $\mathrm{Al}_{2} \mathrm{O}_{3}$ surface. SLG was grown by CVD ${ }^{[17]}$ and then deposited onto the chip via wet transfer ${ }^{[52,53]}$ and subsequently patterned with lithography and reactive ion etching (see the Supporting Information).

Optical Microscopy and Spectroscopy: Optical dark-field images were recorded on a custom-modified Olympus BX51. Samples were illuminated with a focused white light source (halogen lamp). The scattered light was collected through a 100x dark-field objective (Olympus LMPLFLN100xBD, NA 0.8) and analyzed with a fiber-coupled ( $50 \mu \mathrm{m}$ core diameter optical fiber) Ocean Optics QE65000 cooled spectrometer. A standard diffuser was used as a reference to normalize white light scattering. Reflectivity measurements were performed through a $50 \times$ objective (Olympus LMPLFLN100xBD, NA 0.5) using a reduced aperture size.

Atomic Force Microscopy: Topography of the sample was performed using an Asylum Research MFP-3D atomic force microscope in noncontact AC mode. Asylum Research AFM Software version 15 was used for instrument control and data post-processing.

Raman Spectroscopy: Graphene was characterized by Raman spectroscopy after growth, after deposition on devices before measurement, and after activation of devices, through a Renishaw InVia spectrometer equipped with a 100x objective. The Raman spectra in Figure S7 (Supporting Information) were measured at $514.5 \mathrm{~nm}$.

\section{Supporting Information}

Supporting Information is available from the Wiley Online Library or from the author. Source data can be found at https://doi.org/10.17863/ CAM.25258.

\section{Acknowledgements}

D.K. and H.P.A.G.A. contributed equally to this work. The authors acknowledge the financial support from EPSRC grant EP/C060649/1, $\mathrm{EP} / \mathrm{L} 027151 / 1, \quad \mathrm{EP} / \mathrm{G} 037221 / 1, \quad \mathrm{EP} / \mathrm{K} 01711 \mathrm{X} / 1, \quad \mathrm{EP} / \mathrm{K} 017144 / 1$, EP/N010345/1, EP/M507799/1, EP/L016087/1, EPSRC NanoDTC, and ERC grants LINASS320503, Hetero2D, MineGrace, and the EU Graphene Flagship.

\section{Conflict of Interest}

The authors declare no conflict of interest.

\section{Keywords}

graphene, nanoactuation, nanoparticles, plasmonic coupling, resistive switching

Received: April 26, 2018

Revised: June 13, 2018

Published online: July 23, 2018

[1] M. Sitti, in Proc. of the 2001 1st IEEE Conf. on Nanotechnology. IEEE-NANO 2001 (Cat. No.01EX516), Maui, HI, USA 2001, pp. 75-80.

[2] B. F. Toler, R. A. Coutu, J. W. McBride, J. Micromech. Microeng. 2013, 23, 103001

[3] A. Jain, H. Qu, S. Todd, H. Xie, Sens. Actuators, A 2005, 122, 9.

[4] O. Sul, E.-H. Yang, Nanotechnology 2009, 20, 095502.

[5] S. Moya, O. Azzaroni, T. Farhan, V. L. Osborne, W. T. S. Huck, Angew. Chem., Int. Ed. 2005, 44, 4578.

[6] Y. Bar-Cohen, Q. Zhang, MRS Bull. 2008, 33, 173.

[7] K Kaneto, J. Phys.: Conf. Ser. 2016, 704, 012004.

[8] Y. Ke, T. Meyer, W. M. Shih, G. Bellot, Nat. Commun. 2016, 7, 10935.

[9] S. Cho, H. J. Roxana, Rev. Nanosci. Nanotechnol. 2012, 1, 85.

[10] L. Chua, IEEE Trans. Circuit Theory 1971, 18, 507.

[11] D. B. Strukov, G. S. Snider, D. R. Stewart, R. S. Williams, Nature 2008, 453, 80

[12] M. K. Hota, M. K. Bera, B. Kundu, S. C. Kundu, C. K. Maiti, Adv. Funct. Mater. 2012, 22, 4493.

[13] F. Meng, B. Sana, Y. Li, Y. Liu, S. Lim, X. Chen, Small 2014, 10, 277.

[14] C. Chen, S. Gao, F. Zeng, G. S. Tang, S. Z. Li, C. Song, H. D. Fu, F. Pan, J. Appl. Phys. 2013, 114, 014502.

[15] D. S. Jeong, R. Thomas, R. S. Katiyar, J. F. Scott, H. Kohlstedt, A. Petraru, C. S. Hwang, Rep. Prog. Phys. 2012, 75, 076502.

[16] J. J. Yang, F. Miao, M. D. Pickett, D. A. A. Ohlberg, D. R. Stewart, C. N. Lau, R. S. Williams, Nanotechnology 2009, 20, 215201.

[17] X. Li, W. Cai, J. An, S. Kim, J. Nah, D. Yang, R. Piner, A. Velamakanni, I. Jung, E. Tutuc, S. K. Banerjee, L. Colombo, R. S. Ruoff, Science 2009, 324, 1312.

[18] B. de Nijs, R. W. Bowman, L. O. Herrmann, F. Benz, S. J. Barrow, J. Mertens, D. O. Sigle, R. Chikkaraddy, A. Eiden, A. Ferrari, O. A. Scherman, J. J. Baumberg, Faraday Discuss. 2015, 178, 185.

[19] S. Huang, T. Ming, Y. Lin, X. Ling, Q. Ruan, T. Palacios, J. Wang, M. Dresselhaus, J. Kong, Small 2016, 12, 5190.

[20] G. Di Martino, S. Tappertzhofen, S. Hofmann, J. Baumberg, Small 2016, 12, 1334.

[21] A. C. Ferrari, F. Bonaccorso, V. Fal'ko, K. S. Novoselov, S. Roche, P. Bøggild, S. Borini, F. H. L. Koppens, V. Palermo, N. Pugno, J. A. Garrido, R. Sordan, A. Bianco, L. Ballerini, M. Prato, E. Lidorikis, J. Kivioja, C. Marinelli, T. Ryhänen, A. Morpurgo, J. N. Coleman, V. Nicolosi, L. Colombo, A. Fert, M. Garcia-Hernandez, A. Bachtold, G. F. Schneider, F. Guinea, C. Dekker, M. Barbone, Z. Sun, C. Galiotis, A. N. Grigorenko, G. Konstantatos, A. Kis, M. Katsnelson, L. Vandersypen, A. Loiseau, V. Morandi, D. Neumaier, E. Treossi, V. Pellegrini, M. Polini, A. Tredicucci, G. M. Williams, B. H. Hong, J.-H. Ahn, J. M. Kim, H. Zirath, B. J. van Wees, H. van der Zant, L. Occhipinti, A. D. Matteo, I. A. Kinloch, T. Seyller, E. Quesnel, X. Feng, K. Teo, N. Rupesinghe, P. Hakonen, S. R. T. Neil, Q. Tannock, T. Löfwander, J. Kinaret, Nanoscale 2015, 7, 4598.

[22] L. G. Cançado, A. Jorio, E. H. M. Ferreira, F. Stavale, C. A. Achete, R. B. Capaz, M. V. O. Moutinho, A. Lombardo, T. S. Kulmala, A. C. Ferrari, Nano Lett. 2011, 11, 3190.

[23] D. M. Basko, S. Piscanec, A. C. Ferrari, Phys. Rev. B 2009, 80, 165413. 
[24] A. Das, S. Pisana, B. Chakraborty, S. Piscanec, S. K. Saha, U. V. Waghmare, K. S. Novoselov, H. R. Krishnamurthy, A. K. Geim A. C. Ferrari, A. K. Sood, Nat. Nanotechnol. 2008, 3, 210.

[25] T. M. G. Mohiuddin, A. Lombardo, R. R. Nair, A. Bonetti, G. Savini, R. Jalil, N. Bonini, D. M. Basko, C. Galiotis, N. Marzari, K. S. Novoselov, A. K. Geim, A. C. Ferrari, Phys. Rev. B 2009, 79, 205433.

[26] D. Yoon, Y.-W. Son, H. Cheong, Phys. Rev. Lett. 2011, 106, 155502.

[27] A. C. Ferrari, D. M. Basko, Nat. Nanotechnol. 2013, 8, 235.

[28] A. C. Ferrari, J. C. Meyer, V. Scardaci, C. Casiraghi, M. Lazzeri, F. Mauri, S. Piscanec, D. Jiang, K. S. Novoselov, S. Roth, A. K. Geim, Phys. Rev. Lett. 2006, 97, 187401.

[29] X. Cao, X. Li, X. Gao, W. Yu, X. Liu, Y. Zhang, L. Chen, X. Cheng, J. Appl. Phys. 2009, 106, 073723.

[30] G. Ma, X. Tang, H. Zhang, Z. Zhong, X. Li, J. Li, H. Su, J. Mater. Sci. 2017, 52, 238

[31] K. Yan, M. Peng, X. Yu, X. Cai, S. Chen, H. Hu, B. Chen, X. Gao, B. Dong, D. Zou, J. Mater. Chem. C 2016, 4, 1375.

[32] F. Hui, E. Grustan-Gutierrez, S. Long, Q. Liu, A. K. Ott, A. C. Ferrari, M. Lanza, Adv. Electron. Mater. 2017, 3, 1600195.

[33] F. Benz, C. Tserkezis, L. O. Herrmann, B. de Nijs, A. Sanders, D. O. Sigle, L. Pukenas, S. D. Evans, J. Aizpurua, J. J. Baumberg, Nano Lett. 2015, 15, 669.

[34] J. J. Mock, R. T. Hill, A. Degiron, S. Zauscher, A. Chilkoti, D. R. Smith, Nano Lett. 2008, 8, 2245.

[35] T. Ding, C. Rüttiger, X. Zheng, F. Benz, H. Ohadi, G. A. E. Vandenbosch, V. V. Moshchalkov, M. Gallei, J. J. Baumberg, Adv. Opt. Mater. 2016, 4, 877.

[36] C.-Y. Lin, C.-Y. Wu, C.-Y. Wu, C. Hu, T.-Y. Tseng, J. Electrochem. Soc. 2007, 154, G189.

[37] K. M. Kim, B. J. Choi, B. W. Koo, S. Choi, D. S. Jeong, C. S. Hwang, Electrochem. Solid-State Lett. 2006, 9, G343.
[38] L. Chua, Semicond. Sci. Technol. 2014, 29, 104001.

[39] A. G. Radwan, M. E. Fouda, On the Mathematical Modeling of Memristor, Memcapacitor, and Meminductor, Springer, Cham 2015, pp. 13-49.

[40] D. Ielmini, R. Waser, Resistive Switching: From Fundamentals of Nanoionic Redox Processes to Memristive Device Applications, John Wiley \& Sons, Weinheim, Germany 2015.

[41] D. Carta, A. P. Hitchcock, P. Guttmann, A. Regoutz, A. Khiat, A. Serb, I. Gupta, T. Prodromakis, Sci. Rep. 2016, 6, 21525.

[42] Y. V. Khrapovitskaya, N. E. Maslova, Y. V. Grishchenko, V. A. Demin, M. L. Zanaveskin, Tech. Phys. Lett. 2014, 40, 317.

[43] K. M. Ostyn, C. B. Carter, Surf. Sci. 1982, 121, 360.

[44] J. A. Rodriguez, J. C. Hanson, A. I. Frenkel, J. Y. Kim, M. Pérez, J. Am. Chem. Soc. 2002, 124, 346.

[45] D.-H. Kwon, K. M. Kim, J. H. Jang, J. M. Jeon, M. H. Lee, G. H. Kim, X.-S. Li, G.-S. Park, B. Lee, S. Han, M. Kim, C. S. Hwang, Nat. Nanotechnol. 2010, 5, 148.

[46] R. Muenstermann, T. Menke, R. Dittmann, R. Waser, Adv. Mater. 2010, 22, 4819.

[47] A. Kavokin, Ed., Microcavities, Oxford University Press, Oxford, New York 2011.

[48] M. K. Small, W. D. Nix, J. Mater. Res. 1992, 7, 1553.

[49] C. Lee, X. Wei, J. W. Kysar, J. Hone, Science 2008, 321, 385.

[50] M. Berdova, T. Ylitalo, I. Kassamakov, J. Heino, P. T. Törmä, L. Kilpi, H. Ronkainen, J. Koskinen, E. Hæggström, S. Franssila, Acta Mater. 2014, 66, 370.

[51] J. Zabel, R. R. Nair, A. Ott, T. Georgiou, A. K. Geim, K. S. Novoselov, C. Casiraghi, Nano Lett. 2012, 12, 617.

[52] F. Bonaccorso, Z. Sun, T. Hasan, A. C. Ferrari, Nat. Photonics 2010, $4,611$.

[53] F. Bonaccorso, A. Lombardo, T. Hasan, Z. Sun, L. Colombo, A. C. Ferrari, Mater. Today 2012, 15, 564. 